\title{
Experimental study on the forced convective flow in a channel with heated blocks in tandem
}

\author{
Yau-Ming Chen *, Kun-Chieh Wang \\ Department of Mechanical Engineering, National Taiwan University, Taipei, Taiwan 10764, ROC \\ Received 7 February 1996; received in revised form 5 July 1996; accepted 11 September 1997
}

\begin{abstract}
This study experimentally examines the forced convective flow over two sequentially heated blocks mounted on one principal wall of a channel. The experiments, involving mass transfer, were carried out via the naphthalene sublimation technique (NST). By virtue of the analogy between heat and mass transfer, the results can then be converted to determine the heat transfer. In the experiments, the block spacings were set at 2, 4, 6, 8, 12, 16, and 22 and the Reynolds numbers were set at 1300 and $10^{4}$ which correspond to the laminar and the turbulent convective flow cases, respectively. Results show that the Sherwood number increases or decreases monotonically along the block surfaces in the laminar convection cases; while the hump and sharp increase in the Sherwood number can be found in the turbulent convection cases. This is attributed to the reattachment of the separating bubble and the flow impingement, respectively. Comparison between the experimental and numerical results is made and the effect of the block spacing on heat transfer is discussed. (C) 1998 Elsevier Science Inc. All rights reserved.
\end{abstract}

Keywords: Forced convection; Naphthalene sublimation technique; Reattachment; Separating bubble; Flow impingement

\section{Introduction}

The heat transfer and flow characteristics in a channel or duct with heated blocks have in the past been the subject of numerous investigations. This is partly because of its relevance in a multitude of engineering applications and partly because these simple geometries lend themselves well as a proving stone on which theoretical or numerical models can be tested. A better understanding of the detailed flow and thermal characteristics of discrete heated protrusions in forced convection situations is therefore highly desirable from both practical and fundamental points of view. However, the existing literature which shows an insight into the detailed heat transfer and flow behavior is still limited. Previous studies pertinent to this work are briefly summarized below.

Considering the cold flow predictions, many investigations of turbulent recirculating flows in the past have been concerned principally with testing turbulence models, particularly the $k-\epsilon$ model [1-4]. Generally speaking, the $k-\epsilon$ model was found to be a viable alternative to the more complex schemes involving higher or-

\footnotetext{
${ }^{*}$ Corresponding author. Tel.: +8862 3634701; fax: +88623631755.
}

der turbulence closures. Because of its successful performance, as well as its relative simplicity and adaptability, this model is, arguably, the most popular one used in numerical predictions of turbulent flow and heat transfer today. Besides, it is noted that, in the predictions of turbulent convective heat transfer problems, the accuracy of calculation results primarily depend upon the ability to analyze the velocity and the temperature fields in the near wall region. Jones and Launder [5] suggested that, for accuracy and width of applicability, a fine-grid, low-Reynolds-number treatment should be employed near the wall in place of wall functions, despite the attractive simplicity of the latter approach.

Heat transfer predictions for discrete heated protrusions in forced convection situations have also been treated to some extent in the literature. In the laminar forced convection studies, Davalath and Bayazitoglu [6] numerically studied the conjugate heat transfer for the two-dimensional developing flow over a sequence of three rectangular heat generating blocks placed in a parallel plate channel. Similar studies were conducted by Zebib and Wo [7]. Hsieh and Huang [8] studied the separated laminar forced convection near surfacemounted ribs numerically and gave a correlating equation of average Nusselt number as a function of the 
Reynolds number and the rib width. As to the turbulent forced convective flows, only a few studies could be found. Knight and Crawford [9] made a simulation of convective heat transfer in channels with periodically varying cross-sectional area. Wietrzak and Poulikakos [10] carried out a numerical study on the turbulent forced convective cooling of a heated rectangular block. In their work, a low-Reynolds-number version of the $k-\epsilon$ model was used to predict thermal and flow fields. Unfortunately, there was no experimental data for comparison with their numerical predictions.

In the past studies of the flow field measurement, a large proportion of the available experimental work was restricted to the measurement of integral quantities, such as the length of the recirculation region or the mean pressure coefficient. Recently, detailed measurements of the flow field have been carried out by some workers [11-14] who used the Laser-Doppler velocimeter to obtain the velocity distributions and other related quantities.

Similar to the large numbers of studies on the integral quantities found in the flow field measurements, there were also many published papers studying the average heat transfer coefficient for blocks in tandem or problems of the same kind [15-20]. It is worth noting that the traditional technique for measuring the local heat flux and wall temperature distributions with heat flux gages and thermocouples is not only difficult but also inefficient in studies with such a configuration. Sparrow et al. [21-24] made serial investigations on the heat transfer and pressure drop characteristics of arrays of rectangular modules deployed along one wall of a flat rectangular duct via the naphthalene sublimation technique. In their studies, however, only module-averaged heat/mass transfer coefficients were obtained.

The brief literature survey made above clearly indicates the lack of experimental studies on the laminar and the turbulent convective flow over heated blocks in a channel. Even the most basic information such as the local velocity, the turbulent kinetic energy, and the heat transfer coefficient around the heated blocks is lacking. Therefore, the present study attempts to experimentally characterize the laminar and the turbulent convective flows over protruding heated blocks in tandem in a smooth channel. Detailed measurement of heat/mass transfer coefficients were performed using the NST (Naphthalene sublimation technique). Numerical results including both the laminar and the turbulent conditions were also conducted for comparison and understanding of the fluid flow and heat transfer mechanisms.

\section{Experimental apparatus and measuring technique}

The apparatus used in this study is comprised of three major components: the test blocks, the wind tunnel, and the automated data acquisition system. This apparatus was designed to measure the mass transfer coefficient from the surfaces of the blocks.

\subsection{The test blocks}

Each test block was made of chromium coated deep cooled steel with dimensions of $216 \mathrm{~mm} \times 10 \mathrm{~mm} \times 16$ $\mathrm{mm}$. Details of the block geometry can be found in [25]. A recess about $2 \mathrm{~mm}$ deep was machined into the outer surfaces of the block in the region where naphthalene cover was desired. The naphthalene layer extended about $150 \mathrm{~mm}$ near the central portion of the block. The rest of the area adjacent to the ends was left uncoated. The test block served as a part of the four-piece mold. The other pieces of the mold were fabricated to match the test block precisely and its surface was polished to a mirror-like finish. Six thermocouples were used for temperature measurements on the test block. They were installed at the central region of the block and located about $1 \mathrm{~mm}$ below the outer surface of the naphthalene layer. Three thermocouples were positioned along the block and the other three were located around its circumference. These thermocouples monitored the temperature during the wind tunnel runs as well as during the casting processes.

\subsection{The wind tunnel}

The experiments were performed in a suction-type wind tunnel as shown in Fig. 1(a). The entrance section of the tunnel contained a honey-comb flow straightener and dampening screens before a 10 to 1 contraction section. This was followed by a $4 \mathrm{~m}$ long $\times 220 \mathrm{~mm}$ wide $\times 20 \mathrm{~mm}$ high rectangular test section. The first test block was located $2.6 \mathrm{~m}$ downstream from the channel inlet, which corresponds to about 71 hydraulic diameters. Each test block was inserted in a $10 \mathrm{~mm}$ wide $\times 11$ $\mathrm{mm}$ high slot on the bottom surface of the test section. The naphthalene covered portion extended from $2 \mathrm{~mm}$ below the tunnel bottom wall to $5 \mathrm{~mm}$ above it.

The bi-dimensional characteristic of the oncoming flow was confirmed in more than $85 \%$ of the spanwise direction. Flow stability in long duration operations was within $1.5 \%$. The laser Doppler velocimeter was comprised of a $3 \mathrm{~W}$ Argon laser and a transmitting optical arrangement based on an acousto-optic cell. The arrangement is sketched in Fig. 1(b). The optical characteristics of the velocimeter can be found in [25]. The light scattered from the seeding particles was collected in a backward scattering configuration. The Doppler signal was processed by a frequency counter (TSI 1990C) interfaced to a personal computer. The optical components were arranged on a milling table which allowed translation in three directions with accuracy better than $0.1 \mathrm{~mm}$. The corresponding errors were estimated to be within $\pm 2.8 \%$ in the velocity measurement, $\pm 5 \%$ in the turbulence intensity measurement, and $\pm 3.6 \%$ in the Reynolds number measurement.

\subsection{Automated data acquisition system}

The automated data acquisition system was used to measure the local sublimation depth. This system con- 


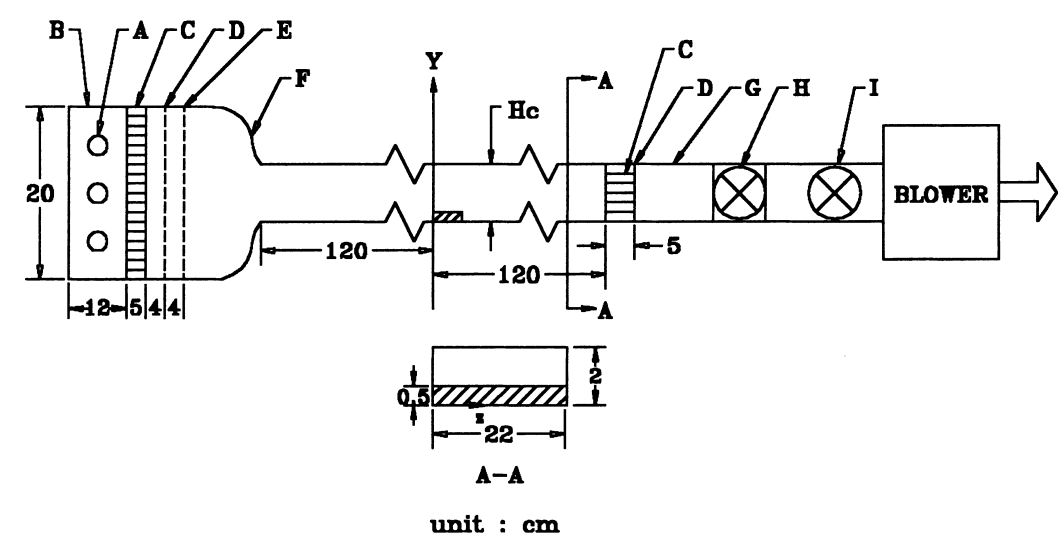

\begin{tabular}{|l|l|l|l|l|l|}
\hline A. & PARTICLE INLET & D. & COARSE SCREEN & G. & CONVERGENT SECTION \\
\hline B. & MLXNG CHAMGER & E. & FINE SCREEN & H. & BY-PASS VALVE \\
\hline C. & HONEYCOMB & F. & CONTRACTION & I & MAIN VALVE \\
\hline
\end{tabular}

(a)Wind tunnel

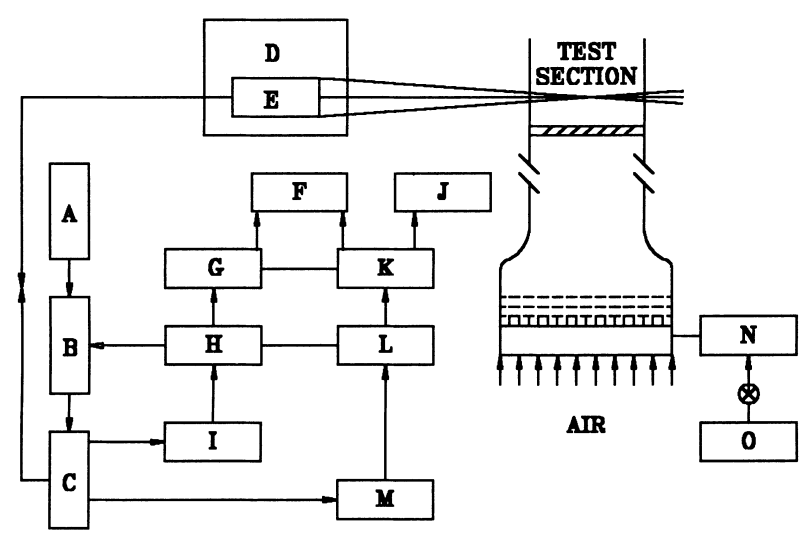

\begin{tabular}{|l|l|c|l|c|l|}
\hline A & AT-LASER & F & OSCIILOSCOPE & K & COUNTER-2 \\
\hline B & COLORBURST & G & COUNTER-1 & L & SHIFTER-2 \\
\hline C & COLOR SEPARATOR & H & SHIFTER-1 & M & PMT-2 \\
\hline D & TRAVELING STAGE & I & PMT-1 & N & JET ATOMISERS \\
\hline E & PROBE & J & PC & O & COMPRESSOR \\
\hline
\end{tabular}

\section{(b)Fiber-LDV system}

Fig. 1. Wind tunnel and fiber-LDV system: (a) Wind tunnel; (b) Fiber- LDV system.

sists of a depth gauge with a signal conditioner, a digital multimeter, two stepping motors, a motor controller unit, and a personal computer. A linear variable differential transformer (LVDT) gauge, having a $2 \mathrm{~mm}$ operation range and $0.1 \mu \mathrm{m}$ resolution, was connected to a signal conditioner. A digital multimeter acquired the signal from the signal conditioner and sent it to the personal computer for data reduction and storage. The personal computer and the motor controller unit controlled the stepping motors to position the LVDT sensor. The stepping motors were able to move the sensor in $1 \mu \mathrm{m}$ increments.

\subsection{Data reduction}

The detailed experimental procedures can be found in [25]. The local mass transfer coefficient is defined as

$h_{\mathrm{m}}=\frac{l_{\mathrm{sb}} \rho_{\mathrm{s}}}{\rho_{\mathrm{nw}}-\rho_{\mathrm{nb}}}$,

where $l_{\mathrm{sb}}$ is the rate of change in naphthalene thickness due to sublimation, $\rho_{\mathrm{s}}$ is the density of the solid naphthalene, $\rho_{\text {nw }}$ is the naphthalene vapor density on the surface, and $\rho_{\mathrm{nb}}$ is the bulk naphthalene vapor density in the channel. The measurement of $l_{\mathrm{sb}}$ is as follows. Before 
experiments, the thickness of the naphthalene cover was measured. After the experiments, the thickness of the naphthalene cover was measured at the same location. The depth of the naphthalene cover due to free convection subtracted from the difference between these two thicknesses is the net sublimation thickness due to forced convection. The $l_{\mathrm{sb}}$ is obtained from this net sublimation thickness divided by the measurement time. The naphthalene vapor density $\left(\rho_{\mathrm{nw}}\right)$ can be determined using the ideal gas law $p=\rho R T$ together with the empirical equation of Ambrose et al. [26]. The results are expressed in terms of the Sherwood number as $\mathrm{Sh}=h_{\mathrm{m}} h / D$, where $h$ is the height of the block and $D$ is the mass diffusion coefficient of naphthalene in the air, which can be determined from Mack's measurement data [27], and as also suggested by Goldstein et al. [28]. The positioning and surface elevation readings using the computer controlled measurement system were found to be very repeatable. Uncertainty analysis according to Kline and McClintock [29] revealed that the estimated errors of the Sherwood numbers were within $7 \%$ in the entire range of our measurements.

\section{Theoretical treatment}

\subsection{Governing equations}

The configuration of the problem under consideration is depicted in Fig. 2. In the laminar convective flow cases, the fluid enters with a parabolic profile from one end and leaves at the other end of the plates carrying the heat dissipated by the blocks. The governing equations used are the steady two-dimensional NavierStokes equations for the incompressible elliptic flow. It is worth noting that, in the energy equation,

$$
U \frac{\partial T}{\partial X}+V \frac{\partial T}{\partial Y}=\frac{\alpha_{\mathrm{s}}}{\alpha_{\mathrm{f}}} \frac{1}{\operatorname{RePr}}\left(\frac{\partial^{2} T}{\partial X^{2}}+\frac{\partial^{2} T}{\partial Y^{2}}\right)
$$

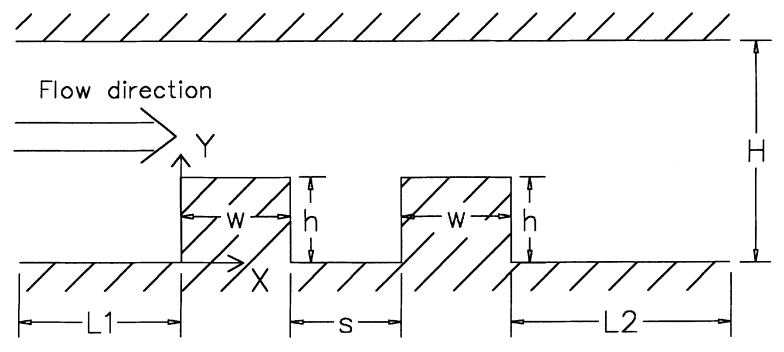

\begin{tabular}{|c|}
\hline$L 1=3 H, h / H=0.25$ \\
\hline$s / h=2 \sim 24, w / h=2$ \\
\hline$L 2=27 H, R e=1300,10000$ \\
\hline
\end{tabular}

Fig. 2. Physical model and configuration of flow field. $\alpha_{\mathrm{s}} / \alpha_{\mathrm{f}}$ means the ratio of thermal diffusivity of a solid block to that of the fluid. In the fluid domain, $\alpha_{\mathrm{s}} / \alpha_{\mathrm{f}}$ is set to one. In the solid region, $\alpha_{\mathrm{s}} / \alpha_{\mathrm{f}}$ is set to a very large value. This makes the terms on the left-hand side of Eq. (2), which represent the thermal convection effects, be neglected and Eq. (2) becomes a simple conduction equation.

In the turbulent convective flow cases, the flow is considered to be steady, incompressible, and two dimensional. The time-averaged Navier-Stokes equation in conjunction with the $k-\epsilon$, low-Reynolds-number closure model proposed by Jones and Launder [5] are used in this study. And, the treatment of $\alpha_{\mathrm{s}} / \alpha_{\mathrm{f}}$ in the energy equation is the same as that in the laminar case.

\subsection{Boundary conditions}

In this study, the inlet conditions of the axial velocity in the laminar convective flow cases; and the axial velocity, the turbulent kinetic energy, and the dissipation rate in the turbulent convective flow cases were obtained by theoretical calculations. This is different from the given semi-empirical or measured inlet conditions which are commonly used in the literature. The adoption of the calculated inlet conditions can reduce the ambiguity coming from the different specifications for the inlet profiles of $U, k$ and $\epsilon$. In the laminar convection cases, the inlet axial velocity is obtained by solving the fully developed forms of the Navier-Stokes equations. The inlet temperature is assumed uniform. In the turbulent convection cases, we first consider an uniform flow in a long smooth parallel channel. Assuming the outlet conditions for $U, k$ and $\epsilon$ are fully developed, the governing equations can be simplified to the fully developed forms. In solving the simplified equations in each iteration, the pressure gradient is set to be able to adjust itself to fit the mass conservation and enable the near wall velocity to satisfy the log-law distribution. After about 200-350 iterations (depending on the Reynolds number), the fully developed profiles for $U, k$ and $\epsilon$ are acquired. The calculated inlet profiles of these variables are shown as an example for $\mathrm{Re}=10^{4}$ in Fig. 3. For comparison, the axial velocity $(U)$, obtained by LDV, is also included. Good agreement can be found between them.

Fluid on the channel walls and block surfaces satisfies the no-slip boundary conditions. Both the upper and the lower walls of the channel were kept adiabatic. The block bases were maintained at a constant temperature $T_{\mathrm{B}}$. Hydrodynamically and thermally fully developed convective flow are assumed at the outlet of the channel. At the fluid-block interface,

$\frac{k_{\mathrm{s}}}{k_{\mathrm{f}}}\left(\frac{\partial T}{\partial n}\right)_{\mathrm{s}}=\left(\frac{\partial T}{\partial n}\right)_{\mathrm{f}} ; \quad T_{\mathrm{s}}=T_{\mathrm{f}}$.

In Eq. (3), the thermal conductivity of block $k_{\mathrm{s}}$ is set to a very large value to ensure constant wall temperature on the block surface. The distance between the inlet and the first block $\left(L_{1}\right)$ is set as $3 H$ and the distance between the second block and the outlet $\left(L_{2}\right)$ is chosen as $27 \mathrm{H}$ in both the laminar and the turbulent convective flow 


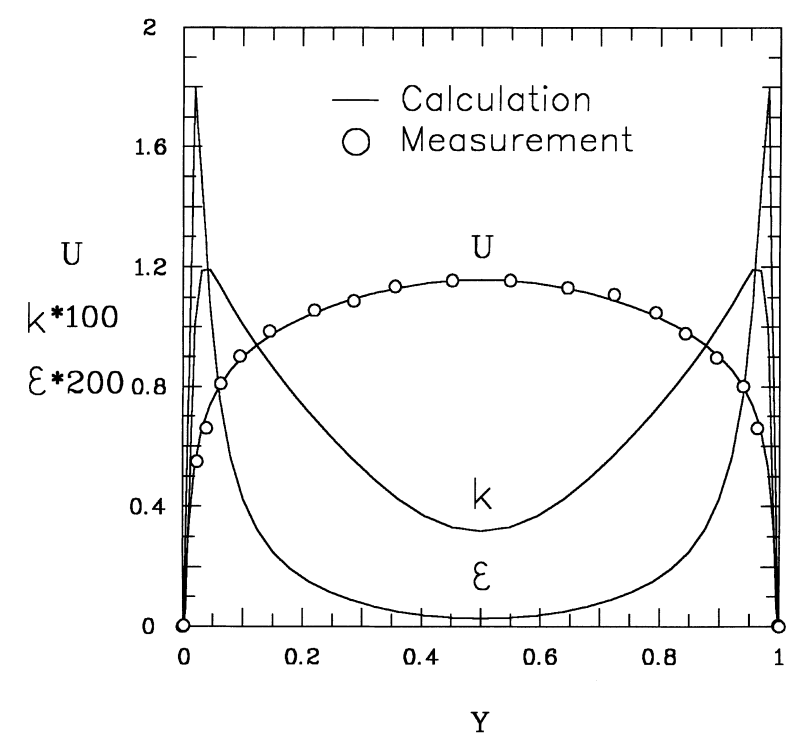

Fig. 3. Inlet profiles of velocity, turbulent kinetic energy, and dissipation rate for $\mathrm{Re}=10^{4}$.

cases. The chosen distance $L_{2}$ is considered to be adequate, since no noticeable change for $\mathrm{Lr}_{2}$ and $\overline{\mathrm{Nu}}$ could be found in the numerical tests as $L_{2}$ is higher than $27 \mathrm{H}$.

\subsection{Numerical scheme}

The governing equations and boundary conditions were made discrete by a control - volume, finite - difference method. The numerical solution was obtained by using the SIMPLER algorithm [30]. The convective and diffusive fluxes were approximated using the power-law scheme which is a more judicious approximation than either a hybrid or an upwind scheme. The discrete conservation equations were solved by the TDMA and the line-by-line iteration method. The use of these schemes was necessary to obtain a faster convergence rate. The under-relaxation factor was carefully chosen to prevent large variations in the source terms. The solution was assumed to be converged when the following criterion was satisfied.

$\frac{\sum_{j=1}^{m} \sum_{i=1}^{n}\left|\phi_{i, j}^{k+1}-\phi_{i, j}^{k}\right|}{\sum_{j=1}^{m} \sum_{i=1}^{n}\left|\phi_{i, j}^{k}\right|} \leqslant \xi$,

where $k+1$ and $k$ respectively denote values in two consecutive iterations, $\phi$ represents the dependent variables of $U, V, P, k, \epsilon$, and $T$; and $\xi$ is a prescribed error. Here, we select $\xi=10^{-4}$ for $\pi=U, V$, and $k$; and $\xi=10^{-5}$ for $\phi=T$. A nonuniform grid was used in both the vertical and the horizontal directions. Tests of various nonuniform grid systems for the laminar convection and the turbulent convection cases were made for $63 \times 21$, $75 \times 27$, and $87 \times 33$; and $140 \times 50,160 \times 70$, and $180 \times$ 90 , respectively. And, the choices of the grid distributions of $75 \times 27$ and $160 \times 70$ are sufficient to provide the grid-independent average Nusselt number for overall faces of the blocks in the laminar and the turbulent con- vection cases, respectively. Further increasing the grid densities to $87 \times 33$ and $180 \times 90$ led to deviations of the overall average Nusselt number with the formers within $1 \%$ and $5 \%$, respectively. The calculations for the laminar and the turbulent convective flows were performed on the PC and the HP computer system (9000 series Model 735), respectively. Typically, it takes about 1000-1500 and 2500-3000 iterations to reach convergence in the laminar and turbulent cases, respectively. The numerical code for the turbulent convective flow cases was tested against previous studies for turbulent flow over a backward facing step [31]. The calculated reattachment length behind the step by this code is $6.5 \mathrm{~h}(\mathrm{~h}$ is the step height) and it is in good agreement with the experimental results as documented in [32]. The heat transfer results are presented in terms of the local $\mathrm{Nu}-$ sselt number at the block surface, defined as

$\mathrm{Nu}=\frac{\left(\frac{\partial \theta}{\partial n}\right)_{\mathrm{w}}}{\theta_{\mathrm{w}}}$

where $n$ is the direction normal to the block surface. The local temperature gradient in Eq. (5) was evaluated by using a three-point Taylor series expansion method.

\section{Results and discussion}

In the experiments, the block spacing $s$ changes from 2 to 24 for both the laminar and the turbulent convective flow cases. For comparison with numerical results, the test conditions for the base cases are at $\mathrm{Re}=1300$ and $s / h=4$ and $\operatorname{Re}=10^{4}$ and $s / h=4$ for the laminar and the turbulent flows, respectively.

\subsection{The streamline and isotherm patterns}

For the laminar convection cases, the calculated flow patterns for $\operatorname{Re}=1300$ and $s / h=4$ are shown in Fig. 4(a). It is seen that there occur separating bubbles on the front and rear faces of the blocks. There is no seperation phenomenon found on the top faces of the blocks. Isotherm patterns shown in Fig. 4(b) demonstrate that dense isotherms occur near the front and the top faces of the blocks, especially near the leading and trailing corners. Better heat transfer can be expected there. On the contrary, sparse isotherms appear near the rear faces of the blocks, indicating worse heat transfer from the blocks there. For the turbulent convection cases, the calculated flow patterns for $\mathrm{Re}=10^{4}$ and $s /$ $h=4$ are demonstrated in Fig. 5(a). Due to the obstruction of the first block, the streamline begins deflection at about $4 h$ upstream from the first block and thus the first separating bubble forms. This bubble is located at the bottom-front corner of the first block, and it has a length of $1.4 \mathrm{~h}$ on the lower wall of the channel and a height of $0.54 h$ on the front face of the first block. As the fluid turns upward into the narrow gap between the top face of the first block and the upper wall of the channel, the fluid is drastically accelerated due to the contraction effect. Meanwhile, because of the large 


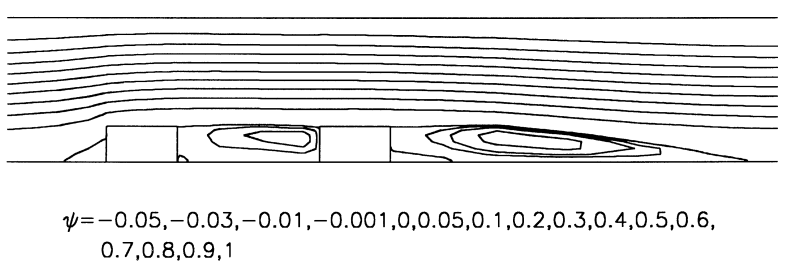

(a) The streamline pattern

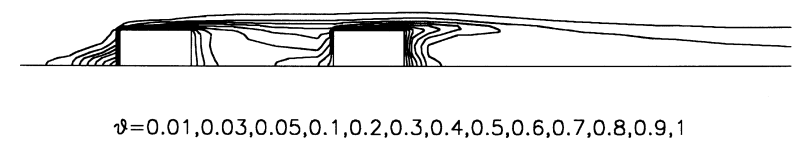

(b) The isotherm pattern

Fig. 4. The streamline and isotherm patterns for $\mathrm{Re}=1300$ and $s / h=4$ : (a) The streamline pattern; (b) The isotherm pattern.

increasing adverse pressure gradient created by the accelerating fluid, the near-wall fluid cannot afford to develop, and thus a small separation bubble is induced. This separating bubble starts at the front corner of the first block, extends along the top face of the first block, and then reattaches to it. This top separating bubble has a reattachment length of $1.1 \mathrm{~h}$. Accompanied by the fluid flow out from the tunnel above the first block, the fluid will directly impinge on the front face of the second block, and there forms a big recirculation region between blocks. It is noted that, on the top face of the sec-

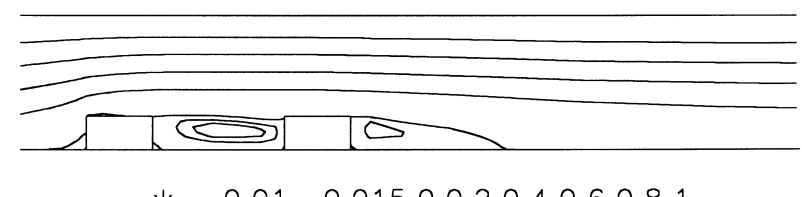

$\psi=-0.01,-0.015,0,0.2,0.4,0.6,0.8,1$

(a) The streamline pattern

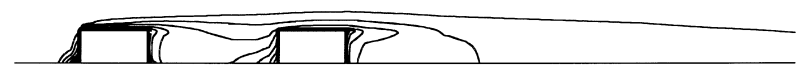

$\Theta=0.1,0.2,0.3,0.4,0.5,0.6,0.7,0.8,0.9,1$

\section{(b) The isotherm pattern}

Fig. 5. The streamline and isotherm patterns for $\mathrm{Re}=10^{4}$ and $s / h=4$ : (a) The streamline pattern; (b) The isotherm pattern. ond block, there is no separating bubble found. Behind the second block, the static pressure increases rapidly due to the expansion effect. The accelerating fluid cannot afford enough axial momentum to overcome the pressure lift in the transverse direction. Thus, a big recirculation forms behind the second block. The reattachment length of this separation region is about $5 h$ and the fluid in it circulates in the clockwise direction. Further downstream, the fluid redevelops and the flow gradually recovers into the unidirectional condition to the outlet of the channel. It requires about a length of $15 \mathrm{~h}$ to attain this fully developed condition. Although the sizes of the existing separating bubbles are all smaller for the turbulent cases than that for the laminar cases, the effects of them on the heat transfer behavior are totally different. This will be discussed later. Isotherm patterns in Fig. 5(b) also indicate similar facts about heat transfer as that found in the laminar case. However, the distribution of more concentrating isotherms around the blocks indicates a much higher heat transfer from the blocks for turbulent convection cases than for laminar ones.

\subsection{Comparison between calculation and LDV measure- ment}

The calculated $U$ and $k$ in the channel with two surface-mounted blocks versus $Y / H$ coordinates at various axial stations are plotted in Fig. 6(a) and (b), respectively. For comparison, the measured $U$ is also depicted. It is worth noting that the isotropic assumption has been employed to obtain the measured $k\left(=1.5 \overline{u^{\prime}}\right)$ since detailed measurements of the turbulence intensity were only made for the axial component. In general, it is seen that the computed results agree well with the measured ones. The negative velocity near the wall around $X=-0.3 H$ indicates the existence of the small separation region at the concave corner of the abrupt contraction formed by the first block. The calculated distance between the separation point and the first block is $1.4 h(0.35 H)$. However, this cannot be confirmed because the seperation zone is too small and too close to the wall to be measured by LDV. Fig. 6(a) indicates that velocity profiles upstream from the first block are faithfully predicted. Over and behind the first and the second block, the calculated axial velocity profiles start to deviate from those measured. The same phenomenon was also indicated by Durst and Rastogi [1]. The calculated negative velocity adjacent to the top wall of the first block indicates the occurrence of flow separation at the convex corner of the abrupt contraction. The distances between the separation point and the reattachment point are $1.1 \mathrm{~h}$ and $1.2 \mathrm{~h}$ according to the computation and the experiment, respectively. In addition, both calculated and measured results show the velocity overshoot near the top face, which comes from the effects of the abrupt contraction. This velocity overshoot is gradually diminishing from $X=0.1 H$ to $X=0.5 H$ due to the viscous effect. Downstream behind the first block, the finding of negative and zero velocities be- 


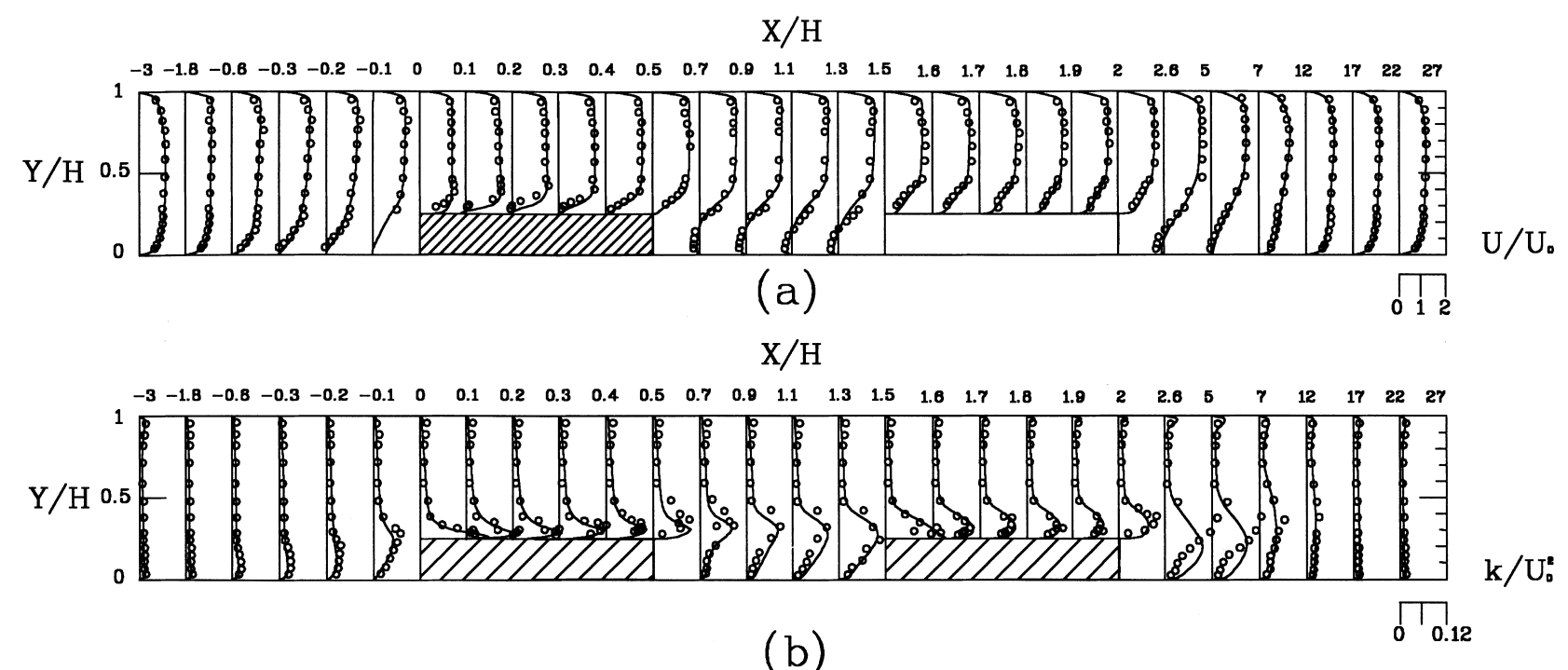

Fig. 6. Distributions of velocity and turbulent kinetic energy along the channel for $\mathrm{Re}=10^{4}$ and $s / h=4$ : (a) distribution of velocity; (b) distribution of turbulent kinetic energy.

tween two blocks indicates the existence of a big recirculation region and the dividing separation line. Upon the top face of the second block, there is no negative velocity found, indicating the absence of the separating bubble. The experimental results also show the same fact. Further downstream behind the second block, the velocity profiles look like that of a backward-facing-step flow. The calculated and measured reattachment lengths are $5.23 h$ and $6.3 h$, respectively. The numerical results seem to overpredict the velocity in the downstream recirculation region behind the second block. After the reattachment point, the flow starts to redevelop and a new developing shear layer is gradually spreading into the old shear layer which originated from the second block. As $X \geqslant 22 H$, the local axial velocity tends to distribute fully developedly till the outlet. The above observations may suggest that the low-Reynolds-number model should be improved in order to achieve a better predition for separated flows.

As to the turbulent kinetic energy distribution $(k)$, from Fig. 6(b), it is seen that $k$ has two local peak values (symmetrical to the centerline) near the channel walls in the inlet section. The peak value of $k$ near the lower wall of the channel gradually increases in the axial direction, and its position gradually shifts towards the front corner of the first block where maximum $k$ occurs. Upon the top face of the first block, the positions of the local maximums at every section above the first block are located just upon the top face. The values of these local maximums are much larger than those before the first block. Between blocks, the local maximums at different axial sections all occur around the separation line. On the top face of the second block, it is found that (1) all peak values for $k$ are smaller than that on the top face of the first block, and (2) the profiles of $k$ are much smoother than that on the top face of the first block. Downstream behind the second block, the local maximums at differ- ent axial sections all occur in the neighborhood of the dividing separation line, just as that behind the first block. After about $X=17 \mathrm{H}, \mathrm{k}$ tends to distribute symmetrically to the centerline, indicating the recovery of the fully developed condition. Overall, the degree of turbulence enhancement is high in the regions around the blocks' corners, the reattachment points, and the separation lines.

Although the quantitative agreement between the calculated and the measured values is not expected, the qualitative agreement is worth noting. The discrepancy between the calculated and measured $k$ occurs mainly in the near wall regions, around the separation lines, and in the separation regions. The calculated peak positions for $k$ are located higher and happen earlier than the measured ones. It may be partly due to the effect of anisotropy in these regions and partly due to the inadequacy of the turbulence model used in the prediction.

\subsection{Comparison between calculation and NST measure- ment}

In the experimental studies what we have obtained is the mass transfer coefficient instead of the heat transfer coefficient. In order to compare the experimental results with the theoretical ones, the analogy between the heat and mass transfer must be used. The Nusselt number distribution can be obtained via the following equation:

$\mathrm{Nu}=\operatorname{Sh}\left(\frac{\mathrm{Pr}}{\mathrm{Sc}}\right)^{n}$

where the power index $n$, according to Igarashi [33], is approximately equal to $1 / 3$. This value has also been used by many other investigators (Goldstein [28]). For laminar convection cases, The Nusselt number distributions along the block surfaces for $\mathrm{Re}=1300$ and $s / h=4$ 


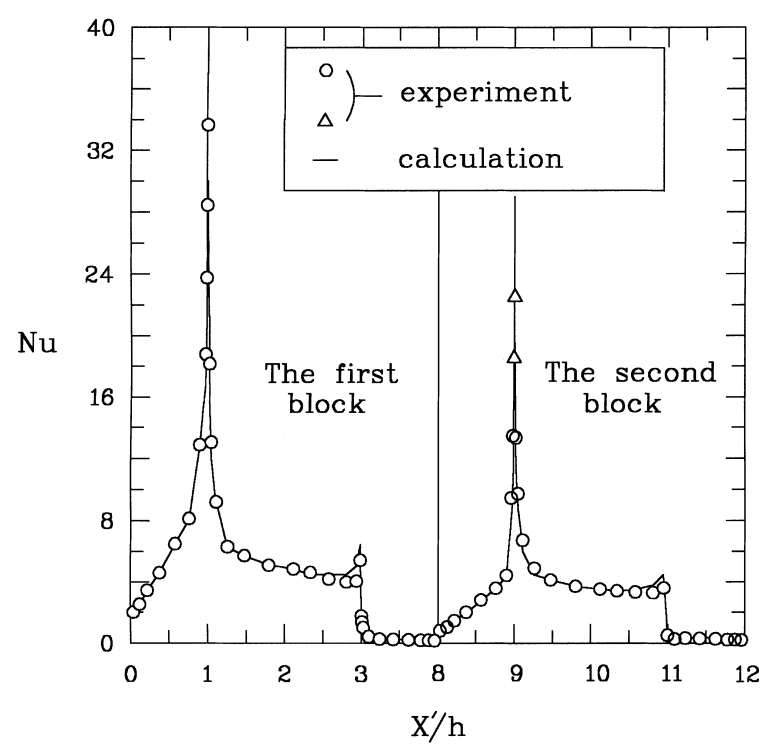

Fig. 7. Comparison between predicted and measured Nusselt number distributions for $\mathrm{Re}=1300$ and $s / h=4$.

are shown in Fig. 7. In this figure, the $X^{\prime}$ coordinate used includes the vertical surfaces of the blocks. Good agreement can be found between the experimental and predicted results. The maximum deviation is less than $9 \%$. The Nusselt number shows a monotonically increasing or decreasing distribution on every face of the two blocks. Due to the strong accelerating and wash-down effects at the front and rear corners of each block, the Nusselt number appears as large and small peaks there, respectively. Besides, the appearance of the separating bubbles on the front face of the first block, between blocks, and behind the rear face of the second block seems to have no apparent contribution to the heat transfer from the blocks.

The local Nusselt number distributions for the turbulent convection case are plotted in Fig. 8. For comparison, the numerical results are also included. As can be seen, good agreement can be found, especially on the front and top surfaces of the two blocks. However, discrepancy does occur on the rear faces of the two blocks. It is worth noting that the discrepancy between heat and mass transfer results on the rear face has also been reported in [25]. In this work, because the agreement between the prediction and experiment on the front and top faces of the two blocks is quite good, the difference occurring on the rear faces might be either attributed to the low-Reynolds-number model used or the analogy equation. The above observations suggest that better understanding of the flow and heat transfer characteristics in a separating and reattaching flow behind the block is essential. From Fig. 8, the Nusselt number humps are found on every face of the two blocks except the front face of the second block where a sharp increase in Nusselt number exists. In comparison to the streamline patterns in Fig. 4, it is seen that (1) for the first block, the reattachment of the separating bubble and

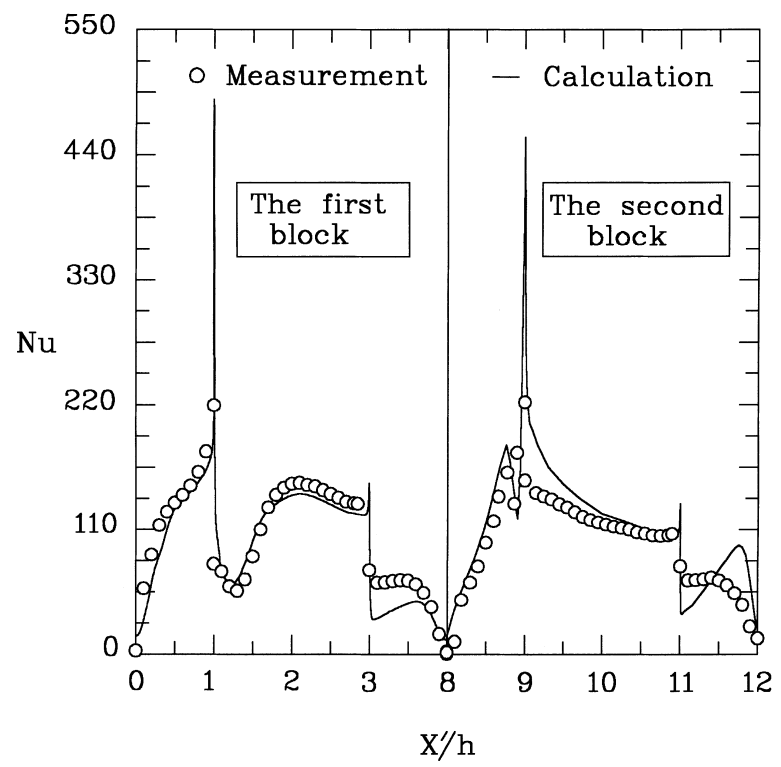

Fig. 8. Comparison between predicted and measured Nusselt number distributions for $\mathrm{Re}=10^{4}$ and $s / h=4$.

the Nusselt number hump both occur at about $0.5 \mathrm{~h}$ and $1.1 \mathrm{~h}$ on the front and top face, respectively; (2) between the two blocks, there exists larger recirculating velocity near the middle section on the rear face of the first block, in which the Nusselt number hump happens nearby. The fluid coming from the tunnel gap near the top wall of the first block directly impinges on the front face of the second block. This impingement causes a sharp increase in the Nusselt number. It is noted that the impingement and the Nusselt number hump are both located near $0.9 \mathrm{~h}$ on the front face of the second block; (3) for the second block, there is no separating bubble found on the top face. The Nusselt number decreases monotonically along the top face. Behind the block, there exists a big recirculation region. The Nusselt number hump occurs around the middle section where larger recirculating velocity exists nearby, just as that on the rear face of the first block.

Overall, the flow impingement and the fluid reattachment are responsible for the occurrence of the sharp increase and hump of the Nusselt number.

\subsection{Local Sherwood number distribution}

\subsubsection{Laminar convective flow}

The Sherwood number distribution along the surfaces of the two blocks for the laminar convection cases $(\mathrm{Re}=1300, s / h=2-24)$ are shown in Fig. 9. All Sherwood number distributions show monotonically increasing or decreasing characteristics on every face of the blocks.

The first block: On the front face, the Sherwood number drastically increases from the front bottom to the maximum at the leading corner. Due to the strong acceleration effect above the reattachment point (about $0.64 h$ ), the slope of the Sherwood number is larger than 


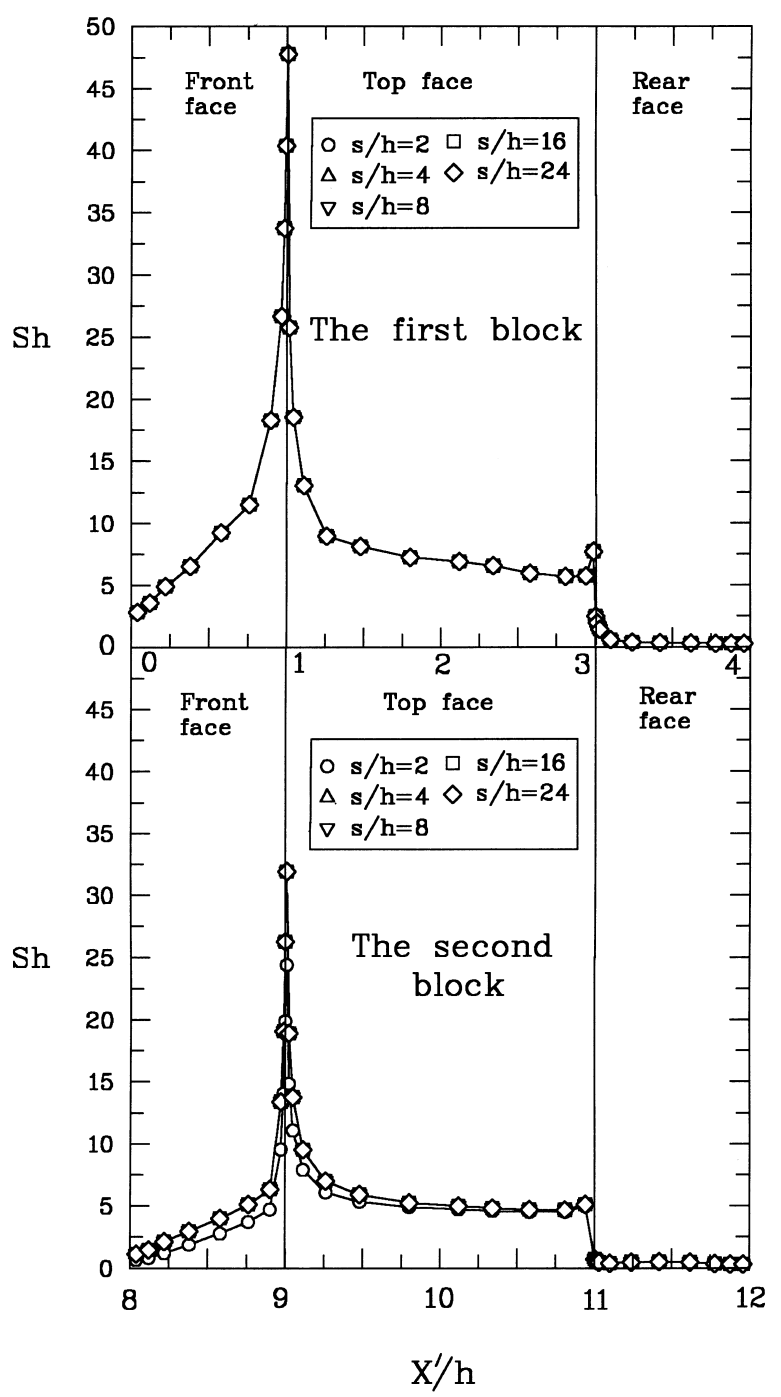

Fig. 9. Effect of the block spacing on the Sherwood number distribution for $\mathrm{Re}=1300$.

that under it. In the neighborhood of the front turning corner, the large contraction effect brings about the occurrence of the strongest accelerating flow and the Sherwood number attains maximum there. Along the top face of the block, the Sherwood number decreases monotonically due to the gradually growing thermal boundary layer. Near the rear corner of the block, the wash-down effect of the fluid induces a slight climb of the Sherwood number. On the rear face of the block, the heat/mass transfer is governed by the cellular motion between blocks. Since the fluid is totally restricted and flows slowly in this area, the Sherwood number decreases first and keeps at a small constant value, which is much smaller than that on the other two faces. The increase in the block spacing seems to have no influence on the distribution of the Sherwood number.

The second block: On every face of the second block, the Sherwood number increases or decreases monotonically, which is similar to but smaller than that of the first block. The smaller values of the Sherwood number are due to less fluid momentum and weaker convective effects around the second block than the first block. Increasing the block spacing only makes a slight increase in the Sherwood number on the front face of the second block.

\subsubsection{Turbulent convective flow}

The Sherwood number distributions along the surfaces of the blocks under different block spacings for $\mathrm{Re}=10^{4}$ are shown in Fig. 10. The major differences in the turbulent convection from the laminar one are the occurrence of the hump and the sharp increase in the Sherwood number which are attributed to the reattachment of separating bubbles and fluid impingement, respectively.

The first block: Along the front face, the Sherwood number increases from the concave corner to the convex corner with a hump in the near-middle section. Accord-

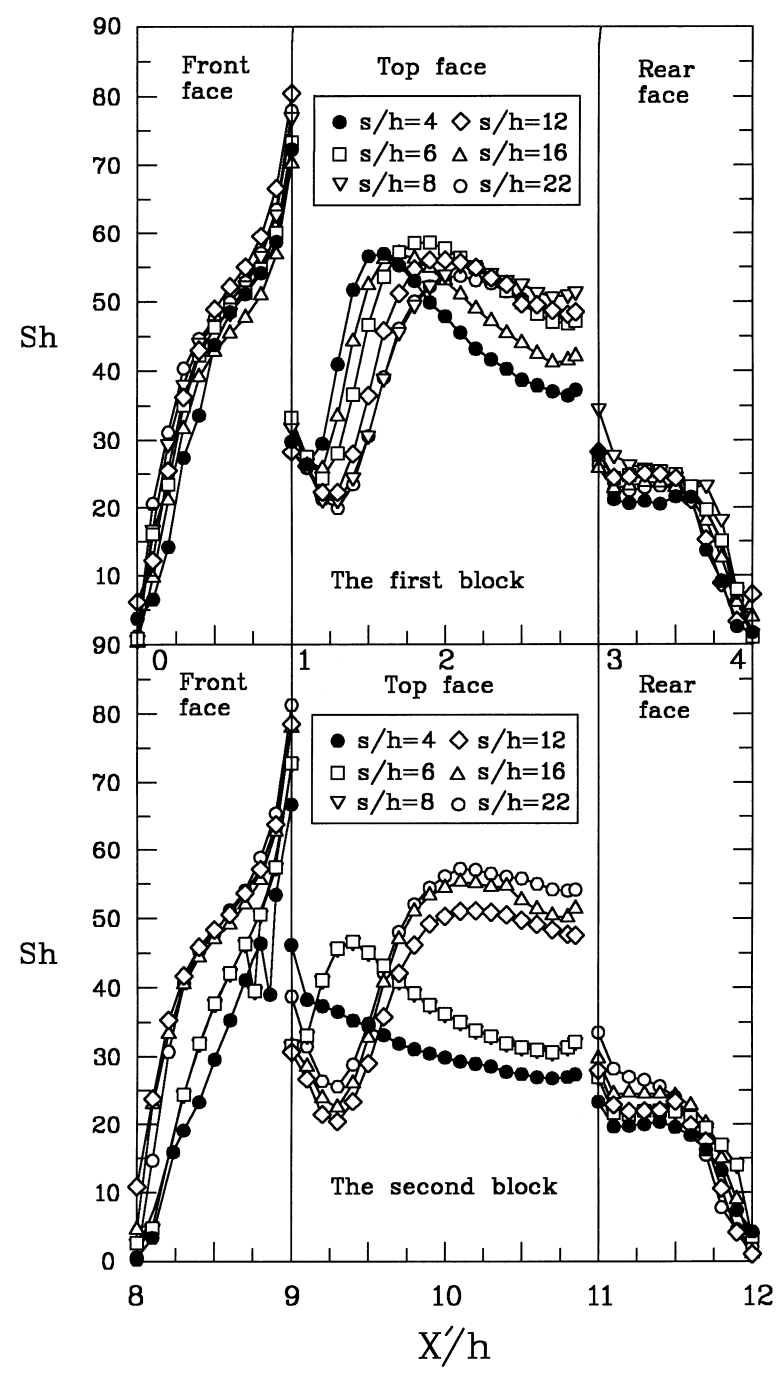

Fig. 10. Effect of the block spacing on the Sherwood number distribution for $\mathrm{Re}=10^{4}$. 
ing to previous discussions, the occurrence of the Sherwood number hump primarily comes from the effect of the flow reattachment of the separating bubble. Examination on the maps at different block spacings indicates that the hump positions as well as the magnitude of the Sherwood number seems to be little affected by the block spacing. Along the top face, all the Sherwood number curves show the same features for different block spacings. The maximum occurs at the leading corner where fluid turns sharply. Downstream there is a low heat transfer rate in the recirculation region, followed by a steep rise to the local maximum near the reattachment point. The Sherwood number hump occurs at the point of reattachment. The reattachment causes a local augmentation of the Sherwood number by a factor of about two, as compared to the local minimum on the same face. Increasing the block spacing from 4 to 22 will lead to about $1 / 5$ fold decrease in the maximum, and about $1 /$ 5 fold decrease in the minimum, and $0.5 \mathrm{~h}$ shift to the right for the Sherwood number hump. On the rear face, the Sherwood number is much less than that on the other two faces. But, there still exists a hump at the middle position due to the recirculating and the fluctuating flow there. The Sherwood number increases slightly with the increasing block spacing.

The Second block: On the top face, the Sherwood number distributions show a great difference between $s / h \geqslant 12$ and $s / h \leqslant 8$. When $s / h \leqslant 8$, the fluid coming from the contraction gap upon the top face of the first block flows along the separation line and impinges on the front face of the second block. This effect leads to a sharp increase in the Sherwood number. Above the impingement point, the Sherwood number slightly decreases because of the slow buffer fluid near this point and then drastically increases to the maximum at the front leading corner because of the strong acceleration effect. As $s / h \geqslant 8$, larger block spacing brings about the occurrence of the Sherwood number hump instead of the sharp increase in the Sherwood number. The distribution of the Sherwood number is qualitatively like that on the front face of the first block. Further increase in the block spacing seems to have no influence on the Sherwood number. On the top face, as $s / h=4$, the block spacing is too small to form a separating bubble. Without the effect of fluctuation and reattachment of the separating bubble, the Sherwood number decreases monotonically as that in the laminar convection cases. As $s / h \geqslant 6$, greater convection effect brings about the occurrence of the top separating bubble. The reattachment of this separating bubble causes the Sherwood number hump, just like that of the first block. The hump position gradually shifts to the right and the Sherwood number gradually increases its value as the block spacing increases. It is noted that, for the second block, the hump positions of the Sherwood number on the top face for $s / h \geqslant 12$ all concentrate near $X^{\prime} / h=10.2$. This means that the top separating bubble of the second block has a constant size as $s / h \geqslant 12$. For the first block, the hump positions of the Sherwood number on the top face scatter around $X^{\prime} / h=1.5-2.0$, indicating that the separating bubble changes with the block spacing. Obviously, the fluid reattachment and flow impingement greatly influence the occurrence, the location, and even the magnitude of the Sherwood number hump in the turbulent convection cases. Comparing the Sherwood number distribution along the top faces of the first and the second blocks under $s / h \geqslant 12$, one finds that they are about the same order but the hump position for the last is located further downstream from the leading corner. On the rear face, the distribution of the Sherwood number is qualitatively and quantitatively like that of the first block. The Sherwood number slightly increases with increasing block spacing.

\subsection{Average Sherwood number}

The average Sherwood number on every face of the block can be obtained by integrating the local values along that face. The results of the average Sherwood number versus the block spacing for the laminar and the turbulent convective flow cases are shown in Fig. 11 and discussed below.

Laminar convection cases: From Fig. 11, for $\mathrm{Re}=1300$, it is seen that $\overline{\mathrm{Sh}_{1}}$ is almost independent of $s / h . \overline{\mathrm{Sh}_{2}}$ and $\overline{\mathrm{Sh}}_{t}$ slightly increases with increasing $s / h$. Changing $s / h$ from 2 to 24 results in an increase of $\overline{\mathrm{Sh}_{2}}$ from the minimum of 3.53 at $s / h=2$ to the maximum of 5.56 at $s / h=24$, about a 1.57 fold increase. For $\overline{\mathrm{Sh}_{t}}$, it increases with increasing $s / h$ from the minimum of 5 at $s / h=2$ to the maximum of 6.17 at $s / h=24$, about a 1.24 fold increase. Obviously, larger block spacing

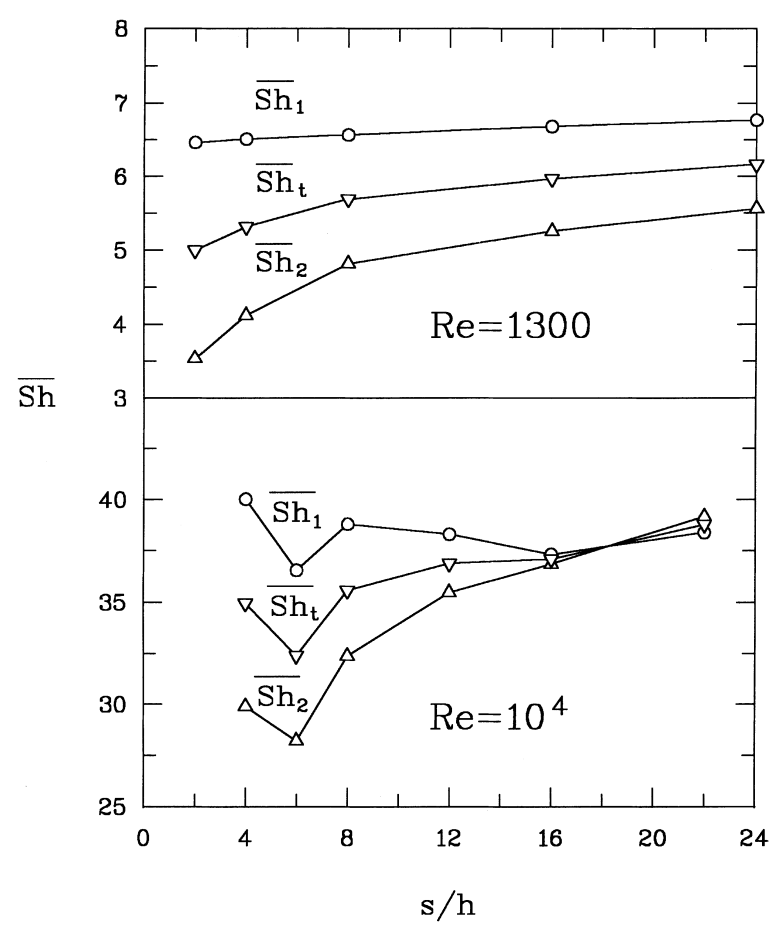

Fig. 11. The average Sherwood number versus the block spacing for $\mathrm{Re}=1300$ and $\mathrm{Re}=10^{4}$. 
brings about better heat/mass transfer from the blocks. An example shows that, for $s / h=8$ and $s / h=16, \overline{S h_{1}}$ are about 1.4 and 1.26 folds of $\overline{\mathrm{Sh}_{2}}$, respectively. $\overline{\mathrm{Sh}_{1}} / \overline{\mathrm{Sh}_{2}}$ is decreasing with $s / h$ from 1.5 to 1.3 and holds at 1.2 as $s / h \geqslant 16$. For a given $\operatorname{Re}$ and $s / h$ say $\mathrm{Re}=1300$ and $s / h=4$, the front face of the first block has the largest average Sherwood number, followed by the top face of the first block, the front face of the second block, the rear face of the second block, and the rear face of the first block. For the total heat/mass transfer amount from the blocks, the front, the top, and the rear faces of the first block and those of the second block contribute sequentially about $24 \%, 40 \%, 1 \%, 7 \%, 27 \%$, and $1 \%$. From the point of view of better heat transfer from the blocks, the Reynolds number and the block spacing should be designed as large as possible.

Turbulent convection cases: From Fig. 11, for $\mathrm{Re}=10^{4}$, it is seen that $\overline{\mathrm{Sh}_{1}}$ rapidly falls down first for $s / h=4-6$, slightly increases for $s / h=6-8$, decreases a little for $s / h=8-16$, and then linearly increases with increasing $s / h$ as $s / h \geqslant 16$. It is noted that the variations of $\overline{\mathrm{Sh}}_{t}$ and $\overline{\mathrm{Sh}}_{2}$ with $s / h$ are somewhat different from that of $\overline{\mathrm{Sh}_{1}}$. As $s / h \leqslant 8$, the distributions of $\overline{\mathrm{Sh}_{1}}, \overline{\mathrm{Sh}_{2}}$, and $\overline{\mathrm{Sh}_{t}}$ are similar to each other. But, as $s / h>8, \overline{\mathrm{Sh}}_{2}$ and $\overline{\mathrm{Sh}_{t}}$ increase with increasing $s / h$. Generally speaking, larger block spacing brings about better heat/mass transfer from the blocks. For the first or the second block, at any fixed block spacing, the average Sherwood number on the front face is in general greater than those on the top and the rear faces. An example shows that, for $s / h=8$ and $s / h=16, \overline{S h_{1}}$ is about 1.2 and 1.02 folds of $\overline{\mathrm{Sh}_{2}}$, respectively. For $\mathrm{Re}=10^{4}$ and $s / h=4$, the front face of the first block has the largest average Sherwood number, followed by the front face of the second block, the top face of the second block, the top face of the first block, the rear face of the second block, and the rear face of the first block. For the total heat/mass transfer amount from the blocks, the above faces contribute sequentially about $17 \%, 32 \%, 8 \%, 13 \%, 24 \%$, and $7 \%$. From the point of view of better heat transfer from the blocks, the Reynolds number and the block spacing should be designed as large as possible.

\section{Practical significance}

The measured local information for fluid flow and heat transfer are of help both in the verification of the numerical codes and in better understanding of the flow and thermal characteristics in such a configuration, which might be applicable to the analysis and design of devices or equipment, such as electronic circuit boards.

\section{Conclusions}

The laminar and the turbulent forced convective flows over heated blocks in tandem in an adiabatic chan- nel have been experimentally and numerically studied. The major conclusions are described as follows:

1. In the laminar convection cases, separating bubbles seem to have no apparent effect on the heat transfer from blocks. The Nusselt number increases or decreases monotonically along the surfaces of the blocks.

2. In the turbulent convection cases, the reattachment of the separating bubbles and the flow impingement are responsible for the occurrence of the hump and the sharp increase of Nusselt number distribution, respectively.

3. In the turbulent convection cases, Detailed comparison of the numerical solution shows good agreement with the LDV measurements near the front and the top recirculation regions. Deviation can be found in the recirculation regions between blocks and behind the second block. It is suggested that the low-Reynolds-number model should be improved in order to achieve a better prediction for separated flows.

4. Comparison between the calculated and the measured Nusselt number distributions show good agreement on all faces of the blocks in the laminar cases and on the front and the top faces of the blocks in the turbulent cases. Deviation can be found on the rear faces of the blocks in the turbulent cases, for which further study is highly needed.

5. The effect of the block spacing on the local heat transfer distribution is remarkable, especially in the turbulent flow cases. Increasing the block spacing lead to a decrease first and then an increase later in the average Nusselt number in the turbulent flow, which is different from the cases in the laminar one.

\section{Nomenclature}

$C_{1} \quad$ model constant

$C_{2}$ model constant

$D$ mass transfer coefficient, $\mathrm{m}^{2} / \mathrm{s}$

$H$ channel height, $\mathrm{m}$

$h$ block height, convective heat transfer coefficient; $\mathrm{m}, \mathrm{J} / \mathrm{s} \mathrm{m}^{2} \mathrm{~K}$

$k \quad$ turbulent kinetic energy, $\mathbf{J}$

$k_{\mathrm{f}} \quad$ thermal conductivity of fluid, $\mathrm{J} / \mathrm{s} \mathrm{m} \mathrm{K}$

$k_{\mathrm{s}} \quad$ thermal conductivity of solid block, $\mathrm{J} / \mathrm{s} \mathrm{m} \mathrm{K}$

$L \quad$ characteristic length, $\mathrm{m}$

$L_{\mathrm{c}} \quad$ channel length, $\mathrm{m}$

$L_{\mathrm{r}} \quad$ reattachment length, $\mathrm{m}$

$\mathrm{Nu}$ local Nusselt number, Eq. (5)

$\mathrm{Re}$ Reynolds number, $U_{0} H / v$

$s \quad$ block spacing, $\mathrm{m}$

$T$ mean temperature, $\mathrm{K}$ or ${ }^{\circ} \mathrm{C}$

$T_{0} \quad$ inlet uniform temperature $\mathrm{K},{ }^{\circ} \mathrm{C}$

$T_{\mathrm{B}} \quad$ temperature at block base, $\mathrm{K}$ or ${ }^{\circ} \mathrm{C}$

$U$ mean horizontal velocity component, $\mathrm{m} / \mathrm{s}$

$U_{0} \quad$ uniform mean horizontal velocity component, $\mathrm{m} / \mathrm{s}$

$V$ mean vertical velocity component, $\mathrm{m} / \mathrm{s}$

$w$ block width, $\mathrm{m}$

$X$ horizontal coordinate, $\mathrm{m}$ 
$X^{\prime} \quad$ horizontal coordinate including block's surfaces, $\mathrm{m}$

$Y \quad$ vertical coordinate, $\mathrm{m}$

\section{Greek letters}

$\alpha_{\mathrm{f}} \quad$ thermal pseudo-diffusivity of fluid, $\mathrm{m}^{2} / \mathrm{s}$

$\alpha_{\mathrm{s}} \quad$ thermal pseudo-diffusivity of solid, $\mathrm{m}^{2} / \mathrm{s}$

$\theta \quad$ mean dimensionless temperature, $T-T_{0} / T_{\mathrm{B}}-T_{0}$

$\epsilon \quad$ dissipation rate of turbulent kinetic energy, $\mathrm{m}^{2} / \mathrm{s}$

\section{Subscripts}

in inlet

f front face of the block

$\mathrm{n}$ direction normal to the block surface

$r \quad$ rear face of the block

$t \quad$ overall faces of blocks

$\mathrm{u}$ top face of the block

w wall

\section{Superscripts}

1 the first block

" the second block

- average

\section{Acknowledgements}

Financial support for this work was provided by the National Science Council of the Republic of China under contract No. NSC 82-0401-E-002-133.

\section{References}

[1] F. Durst, A.K. Rastogi, Theoretical and experimental investigations of turbulent flows with separation, in: F. Durst, B.E. Launder, F.W. Schmidt, J.H. Whitelaw (Eds.), Turbulent Shear Flow, vol. 1, Springer, New York, 1979, pp. 208-219.

[2] A.L. Gosman, E.E. Khalil, J.H. Whitelaw, The calculation of two-dimensional turbulent recirculating flows, in: F. Durst, B.E. Launder, F.W. Schmidt, J.H. Whitelaw (Eds.), Turbulent Shear Flow vol. 1, Springer, New York, 1979, pp. 237-255.

[3] C.C. Chieng, B.E. Launder, On the calculation of turbulent heat transfer downstream from an abrupt pipe expansion, Num. Heat Transfer 3 (1980) 189-207.

[4] A.M. Gooray, C.B. Watkins, W.A. Aung, Two-pass procedure for the calculation of heat transfer in recirculating turbulent flow, Num. Heat Transfer 6 (1983) 423-440.

[5] W.P. Jones, B.E. Launder, The calculation of low-Reynoldsnumber phenomena with a two-equation model of turbulence, Int. J. Heat Mass Transfer 16 (1973) 1119-1130.

[6] J. Davalath, Y. Bayazitoglu, Forced convection cooling across rectangular blocks, J. Heat Transfer 109 (1987) 321-328.

[7] A. Zebib, Y.K. Wo, A Two Dimensional Conjugate Heat Transfer Model for Forced Air Cooling of an Electronic Devices, International Packaging Conference, Orlando, FL, 1985, pp. 2224.
[8] S.S. Hsieh, D.Y. Huang, Numerical computation of laminar separated forced convection on surfaced-mounted ribs, Numerical Heat Transfer 12 (1987) 335-348.

[9] R.W. Knight, M.E. Crawford, Numerical prediction of turbulent flow and heat transfer in channels with periodically varying crosssectional area, in: H.R. Jacobs (Ed.), Proceedings of the National Heat Transfer Conference, ASME, New York, 1, 1988, pp. 669676.

[10] A. Wietrzak, D. Poulikakos, Turbulent forced convective cooling of microelectronic devices, Int. J. Heat and Fluid Flow 11 (1990) $105-112$.

[11] F. Durst, K. Rastogi, Turbulent Flow Over Two-Dimensional Fences, Proceedings of the Second Symposium on Turbulent Shear Flows, Imperical College, London, 1979, pp. 16.30-16.38.

[12] F. Durst, M. Founti, S. Obi, Experimental and computational investigation of the two-dimensional channel flow over two fences in tandem, ASME J. of Fluids Eng. 105 (1988) 48-54.

[13] F. Durst, W.F. Schierholz, Experimental and numerical investigation of plane duct flows with sudden contraction, ASME J. of Fluid Eng. 109 (1987) 376-383.

[14] F. Durst, C. Tropea, Flows over two-dimensional backwardfacing steps, Structure of Complex Turbulent Shear Flow, Springer, Berlin, 1983, pp. 41-48.

[15] G.L. Lehman, R.A. Wirtz, Convection from surface mounted repeating ribs in a channel flow, ASME Paper No. 84-WA/HT$88,1985$.

[16] C.R. Biber, B.G. Sammakia, Transport from discrete heated components in a turbulent channel flow, ASME Paper No. 86WA/HT-68, 1986.

[17] M.J. Chang, R.J. Shyu, L.J. Fang, An experimental study of heat transfer from surface mounted components to a channel airflow, ASME Paper No. 87-HT-75, 1987.

[18] A.B. McEntire, B.W. Webb, Local forced convective heat transfer from protruding and flush-mounted two-dimensional discrete heat sources, Int. J. Heat Mass Transfer 33 (1990) 15211533.

[19] R. Moffat, D.E. Arvizu, The use of superposition in calculating cooling requirements for circuit board mounted electronic components, IEEE Paper No. CH 1781-4/82/0000-B 133.

[20] R. Moffat, D.E. Arvizu, A. Ortega, Cooling electronic components, forced convection experiments with an air-cooled array, Heat Transfer in Electronic Equipment, ASME HTD 48 (1985) $17-27$.

[21] E.M. Sparrow, M. Molki, S.R. Chastain, Turbulent heat transfer coefficients and fluid flow patterns on the faces of a centrally positioned blockage in a duct, Int. J. Heat Mass Transfer 24 (1980) 507-519.

[22] E.M. Sparrow, J.E. Niethammer, A. Chaboki, Heat transfer and pressure drop characteristics of arrays of rectangular modules encountered in electronic equipment, Int. J. Heat Mass Transfer 25 (1982) 961-973.

[23] E.M. Sparrow, S.B. Vemuri, D.S. Kadle, Enhanced and local heat transfer, pressure drop, and flow visualization for arrays of block-like electronic components, Int. J. Heat Mass Transfer 26 (1983) 689-699.

[24] E.M. Sparrow, A.A. Yanezmoreno, D.R. Otis, Convective heat transfer response to height differences in an array of block-like electronic components, Int. J. Heat Mass Transfer 27 (1984) 469473.

[25] J.B. Lin, Heat Transfer of the Horizontal Channel With Blocks in Tandem, M.S. Thesis, National Taiwan University, Taiwan, 1991.

[26] D. Ambrose, I.J. Lawenson, C.H.S. Sprake, The vapor pressure of naphthalene, J. Chem. Thermo. 7 (1975) 1173-1176. 
[27] E. Mack, Jr., Average cross-sectional area of molecules by gaseous diffusion methods, J. Am. Chem. Soc. 47 (1925) 2468 2482.

[28] R.J. Goldstein, S.Y. Yoo, M.K. Chung, Convective mass transfer from a square cylinder and its base plate, Int. J. Heat Mass Transfer 33 (1990) 9-19.

[29] S.J. Kline, F.A. McClintock, Describing uncertainties in singlesample experiments, Mechanical Engineering 75 (1953) 3-8.

[30] S.V. Patankar, Numerical Heat Transfer and Fluid Flow, Hemisphere, New York, 1980.
[31] I.D. Chou, Turbulent Forced Convection in an Adiabatic Channel With a Heated Block, M.S. Thesis, National Taiwan University, Taiwan, 1991.

[32] J.K. Eaton, J.P. Johnston, A review of research on subsonic turbulent flow reattachment, AIAA 19 (1981) 1093-1100.

[33] T. Igarashi, Heat transfer from a square prism to an air stream, Int. J. Heat Mass Transfer 28 (1985) 175-181. 